\title{
Hábitos e estratégias para a realização da lição de casa e variáveis demográficas em estudantes do Ensino Fundamental II
}

\author{
Adriana Cristina Boulhoça Suehiro \\ Universidade Federal do Recôncavo da Bahia, BA, Brasil \\ Evely Boruchovitch \\ Universidade Estadual de Campinas, SP, Brasil
}

\begin{abstract}
Resumo
Descrever os hábitos e estratégias relativas à realização da lição de casa e identificar diferenças em relação a variáveis como idade, estado, gênero e ano escolar foram os objetivos do presente estudo. Participaram 426 alunos, entre 10 e 17 anos, do sexto ao nono ano do Ensino Fundamental, de escolas públicas do interior de São Paulo e da Bahia. Os dados foram coletados mediante um questionário de identificação e uma escala do tipo Likert. Os resultados evidenciaram que os escolares apresentaram bons hábitos e estratégias relativas à lição de casa. Diferenças significativas emergiram somente em relação à idade e ao estado no qual os alunos residiam. Não foram encontradas diferenças significativas em razão do gênero e da progressão escolar.
\end{abstract}

Palavras-chave: Lição de casa; Aprendizagem autorregulada; Estratégias de aprendizagem.

\section{Homework habits and strategies and demographic variables in Elementary School II students}

\begin{abstract}
To describe habits and strategies students employ to do homework and to identify age, state, gender, and school year related differences in these habits and strategies were the objectives of the present study. The sample was composed of 426 students, with ages range between 10 and 17 years, from the sixth to the ninth school year of a public elementary schools in the states of São Paulo and Bahia. Data were collected by means of an identification questionnaire and a Likert type scale. Results showed that students had good habits and strategies for doing homework. Significant differences emerged only for age and state in which students lived. No significant differences were found due to gender and school grade level.
\end{abstract}

Keywords: Homework; Self-regulated learning; Learning strategies.

\section{Hábitos y estrategias para la realización de las tareas y las variables demográficas de los estudiantes de Primaria II}

\section{Resumen}

Describir los hábitos y estrategias relativas a la realización de la tarea y se identifican diferencias en relación a variables como la edad, estado, sexo y año de la escuela eran los objetivos de este estudio. Con la asistencia de 426 estudiantes, entre 10 y 17 años a partir del sexto al noveno año de la escuela primaria, la escuela pública de São Paulo y Bahía. Los datos fueron recogidos mediante un cuestionario de identificación y una escala de Likert. Los resultados mostraron que los estudiantes tenían buenos hábitos y estrategias relacionadas con la tarea. Surgieron diferencias significativas sólo en relación con la edad y el estado en el que los estudiantes vivían. Se encontraron diferencias significativas debidas al género y la progresión de la escuela.

Palabras clave: La tarea; Autorregulado aprendizaje; Estrategias de aprendizaje. 
As pesquisas acerca da aprendizagem revelam que, independentemente do nível de escolarização em que se encontre o estudante, há deficiências de competências necessárias para o aprender com qualidade. Os dados mostram que os alunos não sabem estudar autonomamente e apresentam um nível reduzido de compreensão dos temas estudados (Carvalho, 2012; Pires, 2013; Prates, 2011). Sugerem uma variabilidade no nível de aprendizagem dos escolares em função da presença ou ausência de competências de autorregulação (González, 2013; Paiva \& Lourenço, 2012; Schunk \& Zimmerman, 1994; 1998; Zimmerman, 2008).

A autorregulação é um processo de auto direção, que tem por base o grau de consciência dos indivíduos acerca de suas possibilidades e limitações, do quanto estejam orientados para objetivos ou metas centradas no conhecimento e do domínio de uma gama disponível de estratégias apropriadas (Gomes \& Boruchovitch, 2011; Prates, 2011). Nesse sentido, os hábitos de estudo e as estratégias utilizadas para a realização, por exemplo, da lição de casa, se constituem em fatores relevantes para a compreensão da aprendizagem autorregulada e de qualidade.

A lição de casa, tarefa de casa ou trabalho de casa pode ser definida como uma atividade extraescolar que os estudantes devem realizar no domicilio, com o objetivo de rever e estudar o que aprenderam durante as aulas (Carvalho, 2004; Clement, Custódio, Rufini \& Alves Filho, 2014; Cooper, 2007; Pires, 2013; Sacco \& Boruchovitch, 2014). Embora sua utilização no processo de ensino-aprendizagem ocorra de longa data, não há, entre os teóricos da área, um consenso quanto ao reconhecimento de sua utilidade para o sucesso dos alunos. Há, por um lado, investigadores que defendem a lição de casa como uma atividade essencial para a aprendizagem, pois possibilita a consolidação dos conteúdos aprendidos em contexto da sala de aula e a aquisição de competências para lidar com as dificuldades que surgem durante esse processo (Carr, 2013; Sacco, 2012; Sacco \& Boruchovitch, 2014; Sad \& Gürbüztürk, 2013; Simão, Duarte \& Ferreira, 2012; $\mathrm{Xu}, 2009)$. Por outro, há estudiosos que a consideram algo inútil que retira do estudante tempo para a realização de outras atividades e para o lazer (Araújo, 2006; Marzano \& Pickering, 2007).

Controvérsias a parte, há quem defenda que a lição de casa tem aspectos positivos e negativos (Cooper, Robinson \& Pattal, 2006; Costley, 2013; Marzano \& Pickering, 2007; Pires, 2013; Moreira \& Gabriel, 2013; Simão \& cols., 2012). Sob essa perspectiva, considerase que o aluno que realiza a lição de casa, ao longo do tempo, pode adquirir competências para lidar com as dificuldades que surgem durante o processo de aprendizagem, uma vez que desenvolve gradualmente sua autonomia, aprende a monitorar seu tempo, constrói ou reforça seu sentido de responsabilidade, exercita sua concentração ao lidar com distrações internas e externas e estabelece bons hábitos de estudo (Rosário \& cols., 2005; Sacco, 2012; Sad \& Gürbüztürk, 2013; $\mathrm{Xu}, 2009)$.

De acordo com Boruchovitch (2006), o aluno que é estratégico e autorregulado, na hora de realizar a lição de casa, é capaz de preparar antecipadamente o ambiente em que fará a lição, organizar o material antes de começar as atividades e seu tempo, refazer os exercícios em que encontrou mais dificuldades e não desistir de realizá-la, quando se depara com dúvidas, entre outros aspectos. $\mathrm{O}$ uso das estratégias de aprendizagem, no momento em que se está realizando a lição de casa, é, portanto, de grande relevância, pois colabora para potencializar a aprendizagem. Logo, o aluno que as utiliza, não apenas diversifica as formas de estudo e se auto avalia, mas vai desenvolvendo gradualmente sua autorregulação (Carr, 2013; Oliveira, Boruchovitch \& Santos, 2009; Ramdass \& Zimmerman, 2011).

Embora as estratégias de aprendizagem, no momento em que se está realizando a lição de casa, atuem como importantes reforçadores da aprendizagem, tanto a literatura internacional, quanto a nacional demonstram pouca preocupação com os tipos de estratégias necessários para sua realização (Boruchovitch, 2006; Carr, 2013; Oliveira, Boruchovitch \& Santos, 2009; Ramdass \& Zimmerman, 2011; Sacco, 2012). Nas pesquisas encontradas, discorre-se sobre o importante auxílio dos pais durante a realização da lição de casa, sobre o tempo médio gasto com ela diariamente, a significativa relação entre essa atividade e o desempenho escolar, a necessidade de elaborar lições que estimulem e desafiem o aluno, porém quase não se trata da necessidade de se conhecer e saber como devem ser usadas as estratégias de aprendizagem para a sua otimização (Carvalho, 2006; Ferreira, 2008; Moreira \& Gabriel, 2013; Shumow, 2011).

Do mesmo modo, apesar de as evidências sugerirem que estudantes em anos escolares mais avançados apresentam um melhor repertório de estratégias de aprendizagem, bem como que o sexo feminino tende a ser mais autorregulado quanto à lição de casa, essas variáveis não têm sido alvo das investigações nacionais, sobretudo no que se refere à lição de casa (Vukman \& Licardo, 2010). O único estudo recuperado que verificou essa relação no ensino fundamental foi o de Sacco (2012).

Ao buscar conhecer a opinião dos estudantes a respeito da importância da atividade de lição de casa, investigar o relato de hábitos e uso de estratégias de 
aprendizagem na realização dessa atividade em 280 alunos do terceiro, quinto, sétimo e nono anos do ensino fundamental, Sacco (2012) também verificou as relações entre o relato de hábitos e o uso de estratégias dos estudantes com o seu desempenho escolar, gênero e ano escolar. A maioria dos estudantes avaliados relatou acreditar na importância da lição de casa e os hábitos e estratégias de aprendizagem mais frequentemente referidos foram 'Procurar um lugar bem iluminado para fazer a lição de casa', 'Procurar concentrar-se e prestar atenção quando se está fazendo a lição de casa', 'Acreditar que é capaz de fazer a lição de casa corretamente' e 'Separar todo o material de que precisará, antes de começar a fazer a lição de casa'. Os estudantes de quinto ano obtiveram significativamente as maiores pontuações para o relato de hábitos e o uso de estratégias de aprendizagem, tanto na escala total, quanto no fator composto por estratégias desfavoráveis à aprendizagem. Ao lado disso, constatou-se que, em relação às estratégias benéficas, as diferenças do quinto ano foram significativas somente quando relacionados ao terceiro e ao nono anos, sendo que este último ano obteve a pontuação mais baixa. Os achados de Sacco (2012) revelaram, ainda, que as meninas reportam utilizar mais frequentemente hábitos e estratégias que favorecem a aprendizagem, durante a realização da lição de casa, e que estudantes mais novos têm hábitos e estratégias de aprendizagem significativamente melhores para realizar essa tarefa, quando comparados aos mais velhos.

A despeito do encontrado por Sacco em relação ao preparo para a realização da lição de casa, Pires (2013) verificou que a maior parte dos alunos de sua amostra, 23 estudantes de segundo ano, da cidade de Beja, não planeja suas lições de casa antes de iniciá-las ou não procura/pesquisa qualquer tipo de informação antes de iniciar essa atividade, a não ser que o professor explicite essa necessidade e passe essa orientação. De modo semelhante, a maioria desses escolares realiza as lições de casa de última hora, não avalia seu produto após o término da lição, o que pode indicar, segundo a autora, que eles não sejam capazes de avaliarem seus progressos, e não estejam aptos a gerenciar o seu tempo.

Os achados de Sacco (2012) e Pires (2013) reforçam o fato de que aprender a realizar a lição de casa e a fazer uso de estratégias de aprendizagem e de autorregulação durante essa atividade é fundamental para o bom desempenho nesta tarefa e, consequentemente, para o rendimento escolar do estudante. Diante da importância, dos benefícios e da possibilidade de se desenvolver estratégias de aprendizagem que promovam a autorregulação a partir da lição de casa, bem como da escassez de pesquisas brasileiras com esse olhar e do pouco conhecimento que se tem dos hábitos dos estudantes, o presente estudo teve como objetivo descrever as habilidades e estratégias utilizadas para a realização da lição de casa, de um grupo de estudantes do Ensino Fundamental II, de escolas públicas do interior de São Paulo e da Bahia. Ao lado disso, buscou explorar eventuais diferenças entre os escolares avaliados no que se refere à idade, estado, gênero e ano escolar. Assim, o presente estudo se caracteriza como uma tentativa de somar esforços a investigações como a de Sacco (2012), não apenas para diminuir a lacuna detectada, ao buscar descrever os hábitos e estratégias relativas à realização da lição de casa dos principais atores desse processo, os estudantes, mas também para contribuir para a ampliação do conhecimento acerca dessa importante variável associada ao sucesso escolar.

\section{Método}

\section{Participantes}

Participaram deste estudo 426 estudantes, ambos os gêneros, entre 10 e 17 anos $(M=13,02 ; D P=1,58)$, do sexto ao nono ano do Ensino Fundamental, de escolas públicas do interior de São Paulo e da Bahia. Do total da amostra, 108 escolares $(25,4 \%)$ frequentavam o sexto ano, $105(24,6 \%)$ o sétimo, $110(25,8 \%)$ o oitavo e 103 $(24,2 \%)$ o nono ano, sendo 230 do sexo masculino $(54,0 \%)$ e $196(46,0 \%)$ do feminino. O estado de São Paulo foi representado por 240 alunos $(56,3 \%)$ e a Bahia por 186 (43,7\%).

\section{Instrumentos}

Questões de identificação - consiste em um levantamento de dados sobre algumas características dos participantes: nome, idade, sexo e ano escolar que frequentam, bem como se já repetiram algum ano. Vale lembrar que o nome foi imediatamente substituído por um número de protocolo, conforme recomendação da legislação sobre a ética na pesquisa (Resolução 196/96) com seres humanos.

Escala de Avaliação de Hábitos e Estratégias Relativas à Realização da Lição de Casa (Boruchovitch, 2006) - A "Escala de Avaliação de Hábitos e Estratégias Relativas à Realização da Lição de Casa" tem como objetivo conhecer os hábitos e as estratégias de aprendizagem que os estudantes têm ao realizar a lição de casa. O instrumento possuía, originalmente, 36 itens, em forma de escala likert, redigidos na primeira pessoa do singular. Cada questão oferece três alternativas de escolha ("sempre", "às vezes" e "nunca"). Para a resposta "sempre", foram atribuídos três pontos, para "às vezes", dois pontos, e para "nunca", um ponto, com exceção das questões $1,3,5,7,9,11,13,15,17,20,22,24,26,28$, 
30,33 e 36 , que foram redigidas de forma que sua pontuação, na hora da análise, tenha seu valor invertido. O valor máximo de pontos obtidos na escala era 108 e o mínimo, 36. Quanto maior a pontuação, mais frequente são os bons hábitos e o uso de estratégias de aprendizagem eficientes durante a realização da lição de casa.

Estudos realizados previamente com essa escala (Boruchovitch, 2009), entre 283 estudantes da 3a a $8^{\text {a }}$ séries da educação básica, evidenciaram a alta consistência da escala (Prieto \& Muñiz, 2000), estimada pelo alfa de Cronbach $(\alpha=0,826)$. Resultados do Teste de Esfericidade de Bartlett e do índice de Kaiser-MeyerOlkin (KMO) mostraram ser pertinente a aplicação de análise fatorial na escala. Assim, posteriormente, foi realizada uma análise fatorial exploratória da escala, com rotação varimax, em uma amostra de 646 estudantes do ensino fundamental que apontou uma estrutura de dois fatores. O fator 1 é composto por 15 itens $(1,5,7,9,11,13,15,20,22,24,26,28,30,33$ e 36) que indicam falta de hábitos e estratégias que favorecem a aprendizagem (Ex.: Eu esqueço de fazer a lição de casa.), já o fator 2 contém 15 itens $(2,4,6,8$, 12, 18, 19, 21, 23, 25, 27, 29, 32, 34 e 35) com hábitos e estratégias benéficas à aprendizagem (Ex.: Antes de começar a fazer a lição de casa, eu separo todo o material que vou precisar.). Dos 36 itens originais, seis foram excluídos, por não terem funcionado bem (itens $3,10,14,16,17$ e 31$)$. Tanto a escala total quanto os dois fatores apresentaram consistências internas boas, estimadas pelo alfa de Cronbach, cujos valores foram, respectivamente, 0,759 para a escala total, 0,836 para o fator 1, e 0,802, para o fator 2 (Boruchovitch, 2011).

\section{Procedimento de Coleta de Dados}

Após a aprovação do projeto pelo Comitê de Ética (protocolo 320.889), os instrumentos foram aplicados, coletivamente, em horário de aula previamente cedido pelo professor nas escolas que autorizaram a realização da pesquisa. Inicialmente, os estudantes, cujos pais assinaram o Termo de Consentimento Livre e Esclarecido e que consentiram em participar da pesquisa, preencheram as questões de identificação do sujeito e, após as devidas explicações, foram solicitados a preencherem os itens Escala de Avaliação de Hábitos e Estratégias Relativas à Realização da Lição de Casa. Foram necessários 20 minutos para que todos os procedimentos planejados fossem executados.

\section{Procedimento de Análise de Dados}

Os dados coletados foram analisados em função dos objetivos propostos. Para a caracterização do desempenho dos estudantes no instrumento empregado, foram utilizadas provas de estatística descritiva. Já para a identificação de eventuais diferenças relacionadas a variáveis como os extremos de idade e gênero recorreuse ao teste $t$ de Student. A exploração de diferenças em razão do ano escolar se deu com base na Análise de Variância (ANOVA).

\section{Resultados}

A Tabela 1 apresenta os resultados obtidos pelos alunos na Escala de Avaliação de Hábitos e Estratégias Relativas à Realização da Lição de Casa.

A pontuação total média obtida pelos estudantes avaliados na escala foi de 65,65 pontos $(D P=9,39)$, e a pontuação máxima alcançada foi 87,00 do total de 90 pontos possíveis. Observou-se que o fator 2 (F2), ou seja, Hábitos e Estratégias Benéficos à Aprendizagem alcançou a maior média, o que parece indicar que a amostra apresentou melhores hábitos e estratégias relativas à lição de casa. Os itens mais frequentemente apontados como estratégias que os estudantes utilizam sempre, no fator 2, foram 'Eu acredito que sou capaz de fazer a lição de casa direito' $(n=248 ; 58,2 \%)$, 'Procuro me concentrar e prestar atenção quando estou fazendo a lição de casa' $(n=230 ; 54,0 \%)$, 'Eu procuro um lugar bem iluminado para fazer a lição de casa $(n=224$; $52,6 \%)$ '. 'Eu faço as lições de casa sem ninguém me pedir' $(n=221 ; 51,9 \%)$ e 'Antes de começar a fazer a lição de casa, eu separo todo o material que vou precisar' $(n=220 ; 51,6 \%)$.

Buscando verificar se haveria diferenças entre os participantes em relação à idade, recorreu-se, a análise dos seus extremos, organizando-a em três grupos, até 12 anos; 13 anos e 14 ou mais anos. Foi usado o teste $t$ de Student para a comparação entre os dois grupos constituídos pelos alunos mais novos, situados no quartil 25 - grupo inferior - e o quartil 75 - grupo superior - composto por escolares mais velhos. Evidenciaram-se diferenças significativas entre os alunos mais novos e mais velhos no fator Hábitos e Estratégias Desfavoráveis à Aprendizagem (F1) e na escala Total, tal como pode ser observado na Tabela 2.

Os resultados referentes aos extremos por idade indicaram que os estudantes mais novos apresentaram pontuações médias maiores, quando comparados aos mais velhos, em toda a escala. No que concerne aos seus fatores, observou-se que os estudantes mais novos obtiveram médias significativamente mais elevadas no fator 1, Hábitos e Estratégias Desfavoráveis à Aprendizagem. Os achados concernentes às diferenças por estado estão dispostos na Tabela 3.

As pontuações dos estudantes baianos foram mais altas que a dos paulistas em toda a escala. Foram constatadas diferenças estatisticamente significativas 
entre os estados no fator 2 (F2), ou seja, em Hábitos e Estratégias Benéficos à Aprendizagem (F2) e no Total.

Buscou-se ainda verificar se as pontuações obtidas pelos escolares apresentariam diferenças, ao se considerar variáveis como o gênero e o ano escolar. A Tabela 4 traz os dados relativos ao gênero.

Como pode ser observado na Tabela 4, embora as médias dos meninos tenham sido mais altas que as das meninas no fator 1 (F1), ou seja, em Hábitos e Estratégias Desfavoráveis à Aprendizagem (F1) e no Total, não foram constatadas diferenças estatisticamente significativas entre os gêneros. Do mesmo modo, no que se refere ao ano escolar, a Análise de Variância (ANOVA) não apontou diferenças significativas entre o desempenho dos estudantes em nenhuma medida da escala utilizada. Os índices obtidos foram Hábitos e Estratégias Desfavoráveis à Aprendizagem (F1) $[F(3,422)=0,92 ; p=0,432]$, Hábitos e Estratégias Benéficos à Aprendizagem (F2) $[F(3,422)=0,99$; $p=0,398]$ e Total $[F(3,422)=1,31 ; p=0,272]$. O sétimo e o oitavo ano foram as turmas que apresentaram as menores médias na escala empregada.

TABELA 1

Estatísticas Descritivas das Pontuações Obtidas na Escala de Avaliação de Hábitos e Estratégias Relativas à Realização da Lição de Casa $(\mathrm{N}=139)$

\begin{tabular}{|c|c|c|c|c|}
\hline $\begin{array}{c}\text { Escala de Avaliação de Hábitos e Estratégias Relativas } \\
\text { à Realização da Lição de Casa }\end{array}$ & Mínimo & Máximo & Média & $D P$ \\
\hline Háb. e Estrat. Desfavoráveis à Aprendizagem (F1) & 15,00 & 45,00 & 31,68 & 5,56 \\
\hline Háb. e Estrat. Benéficos à Aprendizagem (F2) & 15,00 & 45,00 & 33,96 & 5,50 \\
\hline Total & 38,00 & 87,00 & 65,65 & 9,39 \\
\hline
\end{tabular}

TABELA 2

Comparação entre as Pontuações Obtidas por Estudantes Mais Novos e Mais Velhos

\begin{tabular}{|c|c|c|c|c|c|c|}
\hline $\begin{array}{c}\text { Escala de Avaliação de Hábitos e Estratégias Relativas } \\
\text { à Realização da Lição de Casa }\end{array}$ & Extremos de Idade & $N$ & Média & $D P$ & $t$ & $p$ \\
\hline \multirow{2}{*}{ Háb. e Estrat. Desfavoráveis à Aprendizagem (F1) } & Até 11 anos & 165 & 32,45 & 6,03 & \multirow{2}{*}{2,50} & \multirow{2}{*}{0,013} \\
\hline & 14 anos ou mais & 163 & 30,89 & 5,20 & & \\
\hline \multirow{2}{*}{ Háb. e Estrat. Benéficos à Aprendizagem (F2) } & Até 11 anos & 165 & 34,45 & 5,67 & \multirow{2}{*}{1,80} & \multirow{2}{*}{0,073} \\
\hline & 14 anos ou mais & 163 & 33,33 & 5,59 & & \\
\hline \multirow{2}{*}{ Total } & Até 11 anos & 165 & 66,90 & 10,14 & \multirow{2}{*}{2,52} & \multirow{2}{*}{0,012} \\
\hline & 14 anos ou mais & 163 & 64,23 & 08,98 & & \\
\hline
\end{tabular}

TABELA 3

Comparação entre as Pontuações Obtidas por Estado $(\mathrm{N}=426)$

\begin{tabular}{|c|c|c|c|c|c|c|}
\hline $\begin{array}{c}\text { Escala de Avaliação de Hábitos e Estratégias Relativas } \\
\text { à Realização da Lição de Casa }\end{array}$ & Estado & $N$ & Média & $D P$ & $t$ & $p$ \\
\hline \multirow{2}{*}{ Háb. e Estrat. Desfavoráveis à Aprendizagem (F1) } & São Paulo & 240 & 31,27 & 5,65 & \multirow{2}{*}{$-1,74$} & \multirow{2}{*}{0,082} \\
\hline & Bahia & 186 & 32,21 & 5,41 & & \\
\hline \multirow{2}{*}{ Háb. e Estrat. Benéficos à Aprendizagem (F2) } & São Paulo & 240 & 33,03 & 5,85 & \multirow{2}{*}{$-4,17$} & \multirow{2}{*}{0,000} \\
\hline & Bahia & 186 & 35,17 & 4,76 & & \\
\hline \multirow{2}{*}{ Total } & São Paulo & 240 & 64,30 & 9,81 & \multirow{2}{*}{$-3,46$} & \multirow{2}{*}{0,001} \\
\hline & Bahia & 186 & 67,39 & 8,55 & & \\
\hline
\end{tabular}

TABELA 4

Comparação entre as Pontuações Obtidas por Gênero $(\mathrm{N}=426)$

\begin{tabular}{|c|c|c|c|c|c|c|}
\hline $\begin{array}{l}\text { Escala de Avaliação de Hábitos e Estratégias Relativas } \\
\text { à Realização da Lição de Casa }\end{array}$ & Gênero & $N$ & Média & $D P$ & $t$ & $p$ \\
\hline \multirow{2}{*}{ Háb. e Estrat. Desfavoráveis à Aprendizagem (F1) } & Masculino & 230 & 32,13 & 5,30 & \multirow{2}{*}{1,80} & \multirow{2}{*}{0,073} \\
\hline & Feminino & 196 & 31,16 & 5,83 & & \\
\hline \multirow{2}{*}{ Háb. e Estrat. Benéficos à Aprendizagem (F2) } & Masculino & 230 & 33,54 & 5,73 & \multirow{2}{*}{$-1,73$} & \multirow{2}{*}{0,084} \\
\hline & Feminino & 196 & 34,46 & 5,18 & & \\
\hline \multirow{2}{*}{ Total } & Masculino & 230 & 65,68 & 9,15 & \multirow{2}{*}{0,07} & \multirow{2}{*}{0,947} \\
\hline & Feminino & 196 & 65,62 & 9,70 & & \\
\hline
\end{tabular}




\section{Discussão}

A lição de casa é uma ferramenta educativa complexa que envolve diferentes atores e contextos. Seu planejamento exige demasiado esmero para que seus objetivos sejam alcançados de forma positiva e para que se constitua, de fato, como uma oportunidade de desenvolvimento da autonomia e de uma série de habilidades importantes para a vida acadêmica de qualquer estudante (Carr, 2013; Sacco, 2012; Sacco \& Boruchovitch, 2014; Sad \& Gürbüztürk, 2013; Simão, Duarte \& Ferreira, 2012; Xu, 2009). Entretanto, pesquisas nacionais na realização da lição de casa que enfoquem o principal ator desse processo, ou seja, o estudante, ainda são restritas. O estudo aqui desenvolvido pretendeu somar esforços aos outros dois localizados (Pires, 2013; Sacco, 2012), se constituir em mais uma contribuição para a compreensão dos recursos empregados pelos alunos durante a realização da lição de casa, desvelando também se há diferenças de gênero, idade, ano escolar, bem como regionais nesta prática.

Assim, constatou-se que os escolares, da amostra pesquisada, obtiveram médias mais elevadas no que diz respeito a Hábitos e Estratégias Benéficos à Aprendizagem, o que sugere que estes estudantes, tal como revelado na pesquisa de Sacco (2012), parecem acreditar na importância da lição de casa, relatando possuir os melhores hábitos e estratégias efetivas relativas à lição de casa. De modo semelhante aos dados de Sacco, entre os itens mais frequentemente apontados como estratégias que os alunos utilizam sempre se encontram 'Eu procuro um lugar bem iluminado para fazer a lição de casa', 'Procuro me concentrar e prestar atenção quando estou fazendo a lição de casa' e 'Antes de começar a fazer a lição de casa, eu separo todo o material que vou precisar'.

Os dados concernentes à idade caminham na mesma direção dos obtidos por Sacco (2012), já que os alunos mais velhos são os que menos reportaram possuir hábitos e estratégias de aprendizagem no momento da realização da lição de casa. Do mesmo modo, sugeriram que os estudantes mais novos têm melhores hábitos e estratégias de aprendizagem para realizar a lição de casa, quando comparados aos mais velhos, uma vez que obtiveram médias mais elevadas no fator 2, Hábitos e Estratégias Benéficos à Aprendizagem (F2), mesmo que essa diferença não tenha sido estatisticamente significativa. Um dado também interessante que o presente estudo possibilitou constatar foi o fato de estudantes baianos terem reportado melhores hábitos e estratégias de aprendizagem para a lição de casa do que os alunos paulistas. Nesse sentido, recomenda-se que futuros estudos possam ser conduzidos em amostras maiores e mais representativas dessas regiões, bem como em outras também.

Diferentemente do verificado por Sacco (2012), não se constataram diferenças estatisticamente significativas entre o desempenho dos estudantes na escala e o ano escolar. Entretanto, independentemente da significância estatística, observou-se que o oitavo e o sétimo ano foram as turmas que apresentaram os piores desempenhos no instrumento. Os resultados aqui obtidos em relação ao ano escolar e à idade chamam a atenção e são preocupantes. Em dissonância com o que aponta a literatura consultada, segundo a qual estudantes em anos escolares mais avançados têm maior probabilidade de disporem de estratégias de aprendizagem mais eficientes e, assim, de uma autorregulação em um estágio mais desenvolvido (Vukman \& Licardo, 2010), os alunos mais velhos e em etapas mais avançadas de escolarização, da amostra estudada, as relatam em menor quantidade e qualidade. Aventa-se aqui como hipótese para o declínio observado o fato de que os estudantes avaliados possam não ter menos ou piores hábitos, mas sim que, possam estar mais desmotivados para relatá-los e/ou usá-los já que evidências provenientes da literatura acerca da variável motivação para aprender revelam o seu decréscimo com o avançar da idade e da escolarização (Baker \& Wigfield, 1999; Gomes \& Boruchovitch, 2013; Monteiro \& Mata, 2001; Rufini, Bzuneck \& Oliveira, 2012). Se por um lado, estudos que incluam essa variável podem ser valiosos para uma maior compreensão do que ocorre com estudantes mais velhos e adiantados na escola, por outro, o dado em si emerge com implicações psicoeducacionais importantes mostrando que esse grupo de alunos deve ser foco de maior atenção e intervenção de professores e educadores.

No que se refere ao gênero, os resultados aqui obtidos indicaram, apesar da ausência de significância estatística, por um lado, que as meninas revelaram utilizar com mais frequência hábitos e estratégias que favorecem a aprendizagem durante a realização da lição de casa, e, por outro, que o gênero masculino relatou, com maior frequência, ter hábitos e fazer uso de estratégias que não são benéficas à aprendizagem. Esses achados corroboram parcialmente os de Sacco (2012) ao se considerar que a autora observou a mesma tendência em relação aos perfis por gênero, no entanto, no estudo dela houve diferença significativa entre meninos e meninas. Considerando as importantes implicações psicoeducacionais que diferenças de gênero possam ter, recomenda-se também que essa variável seja alvo de novas investigações.

Aprender a realizar a lição de casa, mesmo que ela não seja sempre uma atividade prazerosa, é de 
extrema importância, pois as experiências cotidianas com ela oferecem oportunidades para que se possam desenvolver hábitos de trabalho importantes e a utilização de estratégias (Corno \& Xu, 2004; Sacco, 2012, Xu, 2009). Para viabilizar essa prática, no ensino fundamental, os professores podem dar instruções aos alunos sobre como criar hábitos para realizar a lição de casa, como organizar o espaço de trabalho, definir prioridades, controle do tempo e de distrações, ensinar a monitorar emoções indesejadas e reforçar crenças e expectativas de esforço (Carr, 2013; Ramdass \& Zimmerman, 2011).

Em linhas gerais, os dados ora obtidos apresentam importantes implicações educacionais e reforçam o fato de que ensinar aos estudantes como fazer e a se preparar para a lição de casa pode se constituir em um elemento facilitador do processo de aprendizagem e, consequentemente, do sucesso escolar. Sendo assim, espera-se que o presente estudo estimule futuras investigações, de forma a não só neutralizar as limitações desta pesquisa, mas também ampliar o conhecimento sobre os fatores que potencializam e promovem a prendizagem de qualidade de estudantes brasileiros. Tais estudos poderiam lançar mão de amostras maiores e mais representativas, de instituições de natureza jurídica distintas e de regiões diferentes do país, além de incluírem novas variáveis e etapas de escolarização.

\section{Referências}

Baker, L. \& Wigfield, A. (1999). Dimensions of children's motivation for reading and their relations to reading activity and reading achievement. Reading Research Quarterly, 34(4), 452-477. http://dx.doi.org/10.1598/RRQ.34.4.4

Boruchovitch, E. (1999). Estratégias de Aprendizagem e desempenho escolar: considerações para a prática educacional. Psicologia, Reflexão e Crítica, 12(2), 361-376. http://dx.doi.org/10.1590/S0102-79721999000200008

Boruchovitch, E. (2006). Escala de Avaliação de Estratégias Relativas à Realização da Lição de Casa. Manuscrito não publicado. Universidade Estadual de Campinas, São Paulo, SP.

Boruchovitch, E. (2009). Escala de Avaliação de Atitudes Relativas à Realização da lição de casa. In Anais do IV Congresso Brasileiro de Avaliação Psicológica e XIV Conferência Internacional de Avaliação Psicológica: Formas e Contextos e V Congresso Brasileiro de Roscharch e outros Métodos Projetivos (pp. 231-231). Campinas: Universidade São Francisco.

Boruchovitch, E. (2011). Análise fatorial e da consistência interna da escala de hábitos de estudo e estratégias de realização da lição de casa. Relatório técnico não publicado. Faculdade de Educação, Unicamp.

Carr, N. S. (2013). Increasing the Effectiveness of Homework for All Learners in the Inclusive Classroom. School Community Journal, 23(1), 169-182.

Carvalho, M. E. P. (2006). O dever de casa como política educacional e objecto de pesquisa. Revista Lusófona de Educação, 8, 85-102.

Carvalho, M. E. P. (2004). Escola como extensão da família ou como extensão da escola? O dever de casa e as relações família-escola. Revista Brasileira de Educação, 25, 94-104. http://dx.doi.org/10.1590/S1413-24782004000100009

Carvalho, P. S. (2012). Hábitos de estudo e sua influência no rendimento escolar. Dissertação de Mestrado, Universidade Fernando Pessoa, Porto-PT.

Clement, L., Custódio, J. F., Rufini, S. E, \& Alves Filho, J. P. (2014). Motivação autônoma de estudantes de física: evidências de validade de uma escala. Revista Quadrimestral da Associação Brasileira de Psicologia Escolar Educacional, 18(1), 45-56. http://dx.doi.org/10.1590/S1413-85572014000100005

Cooper, H. (1989). Homework. White Plains, NY: Longman. http://dx.doi.org/10.1037/11578-000

Corno, L., \& Xu, J. (2004). Homework as the Job of Childhood. Theory Into Practice, 43(3), 227-233. http://dx.doi. org/10.1353/tip.2004.0032

Ferreira, H. M. (2008). Lição de casa: considerações sobre a relação criançalescrita. Tese de Doutorado, Pontifícia Universidade Católica de São Paulo, São Paulo.

Franco, O. C. de M. (2002). Práticas familiares em relação ao dever de casa: um estudo junto às camadas médias de Belo Horizonte. Dissertação de Mestrado, Universidade Federal de Minas Gerais, Belo Horizonte.

Freire, L. G. L. (2009). Autorregulação da aprendizagem. Ciências e Cognição, 14(2), 276-286.

Frison, L. (2007). Auto-regulação da aprendizagem. Ciência e Conhecimento, 2, 1-14.

Gomes, M. A. M. \& Boruchovitch, (2011). Aprendizagem Autorregulada da Leitura: Resultados Positivos de uma Intervenção Psicopedagógica. Psicologia: Teoria e Pesquisa, 27(3), 291-299.

Gomes, M. \& Boruchovitch, E. (2013b). Motivação para ler e compreensão leitora no Ensino Fundamental-alguns fatores relacionados. In XI CONPE Congresso Nacional de Psicologia Escolar e Educacional (pp. 3-3). Uberlândia: Universidade Federal de Uberlândia.

González, M. L. G. (2013). Learning Goals and Strategies in the Self-regulation of Learning. US-China Education Review A, 3(1), 46-50. http://dx.doi.org/10.1080/13803610903444519 
Maerh, M. \& Meyer, H. (1997). Understanding motivation and schooling: where we've been, where we are and where we need to go. Educational Psychology Review, 9, 399-427.

Monteiro, V. \& Mata, L. (2001). Motivação para a leitura em crianças do 1oㅡ, 2o, $3^{\circ}$ e 4 anos de escolaridade. Infância e Educação - Investigação e Práticas, 3, 49-68.

Moreira, A. S. \& Gabriel, A. P. G. (2013). A importância da participação da família na vida escolar dos alunos dos anos iniciais, segundo professores da escola Nilo Peçanha, do município de Alta Floresta MT. Revista da Faculdade de Alta Floresta (REFAF) (eletrônica), 3(1). Disponível em: http://faflor.com.br/revistas/refaf/index.php/refaf/article/view/110

Oliveira, K. L., Boruchovitch, E., \& Santos, A. A. A. (2009). Estratégias de Aprendizagem e Desempenho Acadêmico: Evidências de Validade. Psicologia: Teoria e Pesquisa, 25(4), 531-536. http://dx.doi.org/10.1590/s010237722009000400008

Paiva, M. O. A. de \& Lourenço, A. A. (2012). A influência da aprendizagem autorregulada na mestria escolar. Estudos e Pesquisas em Psicologia, 12(2), 501-520. http://dx.doi.org/10.12957/epp.2012.8279

Pires, A. F. P. (2013). Os Trabalhos para Casa no 1o Ciclo do Ensino Básico. Relatório de Projeto de Fim de Curso. Escola Superior de Educação do Instituto Politécnico de Beja.

Prates, E. A. R. (2011). Estudo de validade da Escala de Competência em Estudo - ECE-Sup (S\&H) pela correlação com a motivação de universitários. Tese de Doutorado, Universidade São Francisco, Itatiba-SP.

Ramdass, D. \& Zimmerman, B. J. (2011). Developing Self-Regulation Skills: The Important Role of Homework. Journal of Advanced Academics, 22(2), 194-218. http://dx.doi.org/10.1177/1932202X1102200202

Resende, T. F. (2006). Dever de casa: questões em torno de um consenso. In 29ạ Reunião Anual da ANPED: Educação, cultura e conhecimento na contemporaneidade (pp.1-16). Caxambu: Associação Nacional de Pós-Graduação e Pesquisa em Educação.

Rodrigues, R. M. G (1998). Tarefa de casa: um dos determinantes do rendimento escolar. Educação e Filosofia, 12(24), 227-254.

Rosário, P., Mourão, R., Soares, S., Chaleta, E., Grácio, L., Núnez, J. C., \& González-Pienda, J. (2005). Trabalho de casa, tarefas escolares, auto-regulação e envolvimento parental. Psicologia em Estudo, 10(3), 343-351. http://dx.doi. org/10.1590/S1413-73722005000300002

Rufini, S. E., Bzuneck, J. A., \& Oliveira, K. L. (2012). Qualidade da Motivação em Estudantes do Ensino Fundamental. Paidéia, 22(51), 53-62. http://dx.doi.org/10.1590/S0103-863X2012000100007

Sacco, S. G. (2012). Um estudo sobre hábitos e estratégias de aprendizagem na realização da lição de casa de alunos do Ensino Fundamental. Dissertação de Mestrado, Universidade Estadual de Campinas, Campinas-SP.

Schunk, D. \& Zimmerman, B. (1994). Self-regulation of learning and instruction. Hillsdale: Erlbaum.

Schunk, D. H. \& Zimmerman, B. J. (Eds.). (1998). Self-regulated learning: from teaching to self-reflective practice. New York: Guilford Press.

Sad, S. N. \& Gürbüztürk, O. (2013). Primary School Students' Parents' Level of Involvement into their Children's Education. Educational Sciences: Theory \& Practice, 13(2), 1006-1011.

Shumow, L. (2011). Homework and study habits. In S. Redding, M. Murphy, \& P. Sheley (Orgs.). Handbook on family and community engagement (pp. 77-80). Charlotte, NC: Information Age. Disponível em: http://www. schoolcommunitynetwork.org/Default.aspx (see "FACE Handbook").

Vukman, K. B. \& Licardo, M. (2010). How cognitive, metacognitive, motivational and emocional self-regulation influence school performance in adolescence and early adulthood. Eduational Studies, 36, 259-268. http://dx.doi. org/10.1080/03055690903180376

Walberg, H. (1981). A psychology theory of educational productivity. In F. Farley \& N. Gordon. Psychology and education (pp. 81-110). Berkeley: McCutchan.

$\mathrm{Xu}$, J. (2011). Homework completion at the secondary school level: A multilevel analysis. Journal of Educational Research, 104(3), 171-182. http://dx.doi.org/10.1080/00220671003636752

$\mathrm{Xu}$, J. (2009). School location, student achievement, and homework management reported by middle school students. School Community Journal, 19(2), 27-44. Disponível em: http:/www.schoolcommunitynetwork.org/SCJ.aspx

Zimmerman, B. (2008). Investigating self-regulation and motivation: historical background, methodological developments, and future propects. American Educational Research Journal, 45(1), 166-183. http://dx.doi.org/ $10.3102 / 0002831207312909$

Dados dos autores:

Adriana Cristina Boulhoça Suehiro - Doutora, Universidade Federal do Recôncavo da Bahia.

Evely Boruchovitch - Ph.D em Educação, Universidade Estadual de Campinas.

Endereço para correspondência:

Adriana Cristina Boulhoça Suehiro

Rua Rosalvo Fonseca, 225 - São Cristóvão

44571-037 Santo Antônio de Jesus, BA, Brasil

<dricbs@yahoo.com.br>

Recebido em: 22.12.2015

Aceito em: 11.05.2016 